\title{
Aducanumab for Alzheimer's Disease: An Update
}

\author{
Thaarvena Retinasamy and Mohd. Farooq Shaikh*
}

Neuropharmacology Research Strength, Jeffrey Cheah School of Medicine and Health Sciences, Monash University Malaysia, Bandar Sunway 47500 , Selangor, Malaysia.

*Correspondence: farooq.shaikh@monash.edu; Tel.: +603 55144483

Received: 28 June 2021; Accepted: 30 June 2021; Published: 30 June 2021

Edited by: King-Hwa Ling (Universiti Putra Malaysia, Malaysia)

Reviewed by: De Ming Chau (Universiti Putra Malaysia, Malaysia);

Zurina Hassan (Universiti Sains Malaysia, Malaysia)

https://doi.org/10.311187/neuroscirn.v4i2.81

\begin{abstract}
The US FDA approved Aducanumab in June 2021 as the first Alzheimer's disease (AD) drug under its accelerated approval pathway. It has given some hope to patients suffering from AD around the world. Aducanumab is an antibody that targets one of the well-known key culprits of this disease, known as amyloid-beta $(A \beta)$. The journey of Aducanumab was bumpy, and there are controversies around the rapid approval of this drug $A D$ treatment. This article highlights the potential of Aducanumab in AD, its mode of action and controversies around it.
\end{abstract}

Keywords: Alzheimer's disease; amyloid-beta; Aducanumab; Aduhlem; neurodegeneration;

(C2021 by Retinasamy \& Shaikh for use and distribution according to the Creative Commons Attribution (CC BY-NC 4.0) license (https://creativecommons.org/licenses/by-nc/4.0/), which permits unrestricted non-commercial use, distribution, and reproduction in any medium, provided the original author and source are credited.

Alzheimer's disease (AD) is a neurodegenerative disease and also the primary cause of dementia. Approximately 44 million people are living worldwide with $A D$ or a similar form of dementia. By 2050, the number of $A D$ patients may rise from 5.8 million to 13.8 million in America. Additionally, from 2000 to 2018, deaths associated with $A D$ have seen a steep increase of $146.2 \%$, thus making $A D$ the $5^{\text {th }}$ leading cause of death in American geriatrics (2018 Alzheimer's disease facts and figures, 2018).

The clinical expression of $A D$ is believed to begin decades before the onset of the disease, which is observed via the amyloid plaque formation between the neurons, the build-up of intracellular neurofibrillary tau tangles and structural brain changes (Rajan et al., 2015). Plaques and smaller deposits of amyloid-beta $(A \beta)$ termed oligomers trigger neurodegeneration by impeding neuron-to-neuron synaptic communication (Singh et al., 2016). On the other hand, tau tangles are known to obstruct the nutrients and essential molecules required for neuronal survival (Kent et al., 2020). Additionally, both $A \beta$ and tau activate microglia, which triggers neuroinflammation, resulting in impaired glycolysis linked to acute $A \beta$, and tau observed postmortem (An et al., 2018). Three distinct phases in $A D$ development start as a preclinical phase characterised by normal cognition and irregular brain biomarkers, followed by the mild cognitive impairment phase and the clinically apparent dementia phase (Marasco, 2020).

Over the past decade, the therapeutic approaches to $A D$ can be generally divided into two categories, symptomatic treatments, and disease-modifying treatments. Despite significant efforts and international clinical drug trials searching for a potential treatment for AD, only four cholinesterase inhibitors and an NMDA receptor antagonist exerted appropriate safety and efficacy, enabling international endorsement (Farrimond et al., 2012). However, these agents are symptomatic treatments that momentarily improve cognitive problems. Their clinical effects also appeared 
to be modest, and they were unable to resolve the underlying cause of $A D$, thus failed to delay the rate of decline (Cummings et al., 2016; Cummings et al., 2014; Schneider et al., 2014). Recently, the focus on drug discovery and development has moved towards disease-modifying treatments that emphasise the underlying disease pathology by targeting changes observed in the brain of AD patients. These treatments slow down the disease progression and/or impedes its onset (Siemers, 2015). Several candidate agents have been developed; however, they have been unsuccessful in clinical stages and never passed phase 3 clinical trials (Cummings et al., 2016; Siemers, 2015).

The antibody-based immunotherapeutic approach has recently been developed by choosing human B-cell clones that were activated $A \beta$ aggregates epitopes (Sevigny et al., 2016). When the collection of human memory $B$ cells were selected for reactivity against aggregated $A \beta$, Aducanumab, a human monoclonal antibody that was selectively reactive with $A \beta$ aggregates and with soluble oligomers as well as insoluble fibrils, were selected as lead drug candidates (Ferrero et al., 2016). In preclinical studies, aducanumab could cross the blood-brain barrier, which diminished brain amyloid burden (Sevigny et al., 2016). Aducanumab is an antibody that binds to the $A \beta$ aggregates and also the parenchymal amyloid (Crehan \& Lemere, 2016). In preclinical studies using Tg2576 mice, intraperitoneally injected Aducanumab bind and helped in the clearance of parenchymal plaques preventing micro haemorrhages (Dunstan et al., 2011). Additionally, the build-up of brain macrophages surrounding the remaining plaques was also reported, thus insinuating the possibility of phagocytosis being used to remove the $A \beta$ plaques, thus potentially slowing neurodegeneration and disease progression (Crehan \& Lemere, 2016).

Aducanumab, marketed as Aduhlem, is the first $A D$ treatment approved by the U.S. Food and Drug Administration (FDA) since 2003 and this disease's first targeted therapy. This is deemed the first approved treatment to treat AD's root cause instead of just treating the symptoms. FDA gave the green light to the therapy in June 2021 under its accelerated approval pathway that provides early access to this potentially valuable treatment. The accelerated approval regulations allow drugs of severe conditions that filled an unmet medical need to be approved based on a surrogate endpoint. While most drug trials measure success based on clinical endpoints that ascertain whether a drug provides a clinical outcome, a surrogate endpoint is a marker or a measure that is used to predict clinical outcomes. These surrogate endpoints are viable substitutes for hard clinical endpoints because they are linked to the desired clinical outcomes. In the case of Aducanumab, the desired clinical endpoint was to observe a reduction in clinical decline. Since the clinical endpoint was not met, the surrogate endpoint, which reduces amyloid-beta plaque, was considered in approving this drug. However, the accelerated approval pathway requires the company to verify clinical benefits in a post-approval trial. If clinical benefits cannot be verified, the FDA may initiate proceedings to withdraw the approval. This drug approval serves as a positive step forward for patients with Alzheimer's disease who now have a critical new treatment to help combat this disease. Many patients, their families and advocacy groups have extended their support in the approval, stating that Aducanumab is the first to tackle the underlying root cause of $A D$. Although it might not be the cure, it could serve as a start for more enhanced treatments and foster more significant innovation in $A D$ treatment (Mullard, 2021).

Numerous controversies were surrounding the approval of Aducanumab. Although Aducanumab reduced amyloid plaque, there was insufficient validation to prove that this decrease led to clinical significance enhancement of cognitive tests scores (Mullard, 2021). Cognitive tests are accurate assessments of cognitive function and are extremely important in Alzheimer's disease drug development. Since Aducanumab did not demonstrate a reduction in clinical decline depicted as improvement in memory which is crucial in most Alzheimer's drug trials and only showed a reduction in $A \beta$ plaques, the reliability of this drug appeared to be questionable. Furthermore, decreased amounts of plaques even in early $A D$ conditions failed to stop disease progression.

Additionally, post-mortem studies of $A D$ patients in the Elan trial revealed that even though low amounts of amyloid plaque burden were observed, most patients still succumb to AD (Bachmann et al., 2019). Similarly, some monoclonal antibodies (Bapineuzumab, Solanezumab, and Gantenerumab) also reported decreased plaque burden but were unsuccessful in slowing down disease progression (Sevigny et al., 2016). ENGAGE and EMERGE were two identical global Phase 3 randomised, double-blind, placebo-controlled, parallelgroup studies carried out to evaluate the efficacy and safety of Aducanumab in patients with mild cognitive impairment (MCl) due to Alzheimer's disease and mild Alzheimer's disease dementia (Huang et al., 2020; 
Schneider, 2020). During both the studies, participants were randomly assigned either a placebo, low dose of Aducanumab ( $3 \mathrm{mg} / \mathrm{kg}$ if an APOE $\varepsilon 4$ carrier $\& 6 \mathrm{mg} / \mathrm{kg}$ if not) and a high dose of Aducanumab (6 mg/ $/ \mathrm{kg}$ if an $A P O E \& 4$ carrier \& $10 \mathrm{mg} / \mathrm{kg}$ if not), based on their APOE $\varepsilon 4$ genotype which is a key genetic risk factor for $A D$ (Schneider, 2020). The performance of the participants in a battery of cognitive tests served as the primary trial endpoints. In December 2018, both the studies went through a futility analysis; a test used to evaluate if the trial is worth continuing to completion based on preliminary data (Schneider, 2020). The analysis found no benefit versus the placebo at either the low or high doses. Both of these extensive studies were unable to show the drug's effectiveness. However, further analysis of additional data was found to be conflicting, especially the efficacy between the two trials, with only one, EMERGE, showing a benefit in a sub-analysis of data limited to the higher doses (Cummings et al., 2021; Knopman et al., 2021).

Another controversial issue surrounding Aducanumab was the clinical trials conducted on the early to mild stages of $A D$ with evidence of amyloid plaques and were never tested on those with moderate to severe $A D$ patients. However, the drug is not limited to the specific AD stage, which was tested and approved to be widely used for $A D$ in general. Additionally, the data also demonstrated that Aducanumab had significant side effects. In both trials, approximately $40 \%$ of the treated participants developed brain swelling and tiny brain bleeds (Mullard, 2021). Some of the other effects observed include amyloid-related imaging abnormalities (ARIA), which represented outflow via the blood-brain barrier (ARIA-E) or haemorrhages (ARIA-H) linked with a defective blood-brain barrier. It is said that ARIA-E were observed in $34 \%$ and $35.5 \%$ of those receiving high-dose Aducanumab in EMERGE and ENGAGE trials, respectively (Cummings et al., 2021). Likewise, those who proceed to take this treatment will still need to undergo routine brain scans to avoid serious complications, thus exerting a burden on patients, neurologists, and the healthcare system.

Given that there is currently no approved diseasemodifying treatment available for $A D$, it is understandable that most are in a desperate search for one that will delay the onset or reduce the progression of this devastating disease. However, any treatment made available must be beneficial and not unleash a risky precedent that could derail the work being done to develop an effective treatment for AD. Furthermore, approval of a drug or treatment that is not effective has serious potential to impair future research into new medicines that may effectively treat AD. Thus, if Aducanumab turns out to be not as effective, this could, in turn, lower the bar for future drugs and fewer effective therapies.

\section{Acknowledgements: Not Applicable.}

Author Contributions: MFS and TR conceived the idea and TR wrote the paper. MFS provided critical inputs and edited the manuscript.

Conflicts of Interest: The authors declare no conflict of interest.

\section{References}

2018 Alzheimer's disease facts and figures. (2018). Alzheimer's \& Dementia, 14(3), 367-429. https://doi.org/10.1016/j.jalz.2018.02.001

An, Y., Varma, V. R., Varma, S., Casanova, R., Dammer, E., Pletnikova, O., Chia, C. W., Egan, J. M., Ferrucci, L., Troncoso, J., Levey, A. I., Lah, J., Seyfried, N. T., Legido-Quigley, C., O'Brien, R., \& Thambisetty, M. (2018). Evidence for brain glucose dysregulation in Alzheimer's disease. Alzheimer's \& Dementia, 14(3), 318-329. https://doi.org/10.1016/j.jalz.2017.09.011

Bachmann, M. F., Jennings, G. T., \& Vogel, M. (2019). A vaccine against Alzheimer's disease: anything left but faith? Expert Opinion on Biological Therapy, 19(1), 73-78. https://doi.org/10.1080/14712598.2019.1554646

Crehan, H., \& Lemere, C. A. (2016). Chapter 7 - Anti-Amyloid- $\beta$ Immunotherapy for Alzheimer's Disease. In M. S. Wolfe (Ed.), Developing Therapeutics for Alzheimer's Disease (pp. 193-226). Academic Press. https://doi.org/10.1016/B978-0-12802173-6.00007-1

Cummings, J., Aisen, P., Lemere, C., Atri, A., Sabbagh, M., \& Salloway, S. (2021). Aducanumab produced a clinically meaningful benefit in association with amyloid lowering. Alzheimer's Research \& Therapy, 13(1), 98. https://doi.org/10.1186/s13195-021-00838-z

Cummings, J., Aisen, P. S., DuBois, B., Frölich, L., Jack, C. R., Jones, R. W., Morris, J. C., Raskin, J., Dowsett, S. A., \& Scheltens, P. (2016). Drug development in Alzheimer's disease: the path to 2025. Alzheimer's research \& therapy, 8(1), 39. https://doi.org/10.1186/s13195-016-0207-9

Cummings, J. L., Morstorf, T., \& Zhong, K. (2014). Alzheimer's disease drug-development pipeline: few candidates, frequent failures. Alzheimer's research \& therapy, 6(4), 37. https://doi.org/10.1186/alzrt269 
Dunstan, R., Bussiere, T., Fahrer, D., Quigley, C., Zhang, X., Themeles, M., Engber, T., Rhodes, K., Arastu, M., \& Li, M. (2011). Quantitation of beta-amyloid in transgenic mice using whole slide digital imaging and image analysis software. Alzheimer's \& Dementia, 7(4, Supplement), S700. https://doi.org/10.1016/i.jalz.2011.05.2024

Farrimond, L. E., Roberts, E., \& McShane, R. (2012). Memantine and cholinesterase inhibitor combination therapy for Alzheimer's disease: a systematic review. BMJ Open, 2(3), e000917. https://doi.org/10.1136/bmjopen-2012-000917

Ferrero, J., Williams, L., Stella, H., Leitermann, K., Mikulskis, A., O'Gorman, J., \& Sevigny, J. (2016). First-in-human, doubleblind, placebo-controlled, single-dose escalation study of aducanumab (BIIB037) in mild-to-moderate Alzheimer's disease. Alzheimer's \& Dementia, 2(3), 169-176. https://doi.org/10.1016/i.trci.2016.06.002

Huang, L.-K., Chao, S.-P., \& Hu, C.-J. (2020). Clinical trials of new drugs for Alzheimer disease. Journal of Biomedical Science, 27(1), 18-18. https://doi.org/10.1186/s12929-019-0609-7

Kent, S. A., Spires-Jones, T. L., \& Durrant, C. S. (2020). The physiological roles of tau and A $\beta$ : implications for Alzheimer's disease pathology and therapeutics. Acta Neuropathologica, 140(4), 417-447. https://doi.org/10.1007/s00401-020$\underline{\text { 02196-w }}$

Knopman, D. S., Jones, D. T., \& Greicius, M. D. (2021). Failure to demonstrate efficacy of aducanumab: An analysis of the EMERGE and ENGAGE trials as reported by Biogen, December 2019. Alzheimer's \& Dementia, 17(4), 696-701. https://doi.org/https://doi.org/10.1002/alz.12213

Marasco, R. A. (2020). Current and evolving treatment strategies for the Alzheimer disease continuum. American Journal of Managed Care, 26(8 Suppl), S167-s176. https://doi.org/10.37765/ajmc.2020.88481

Mullard, A. (2021). Landmark Alzheimer's drug approval confounds research community. Nature, 594(7863), 309-310. https://doi.org/10.1038/d41586-021-01546-2

Rajan, K. B., Wilson, R. S., Weuve, J., Barnes, L. L., \& Evans, D. A. (2015). Cognitive impairment 18 years before clinical diagnosis of Alzheimer disease dementia. Neurology, 85(10), 898. https://doi.org/10.1212/WNL.0000000000001774

Schneider, L. (2020). A resurrection of aducanumab for Alzheimer's disease. The Lancet Neurology, 19(2), $111-112$. https://doi.org/10.1016/S1474-4422(19)30480-6

Schneider, L. S., Mangialasche, F., Andreasen, N., Feldman, H., Giacobini, E., Jones, R., Mantua, V., Mecocci, P., Pani, L., Winblad, B., \& Kivipelto, M. (2014). Clinical trials and late-stage drug development for Alzheimer's disease: an appraisal from 1984 to 2014. Journal of Internal Medicine, 275(3), 251-283. https://doi.org/10.1111/joim.12191

Sevigny, J., Chiao, P., Bussière, T., Weinreb, P. H., Williams, L., Maier, M., Dunstan, R., Salloway, S., Chen, T., Ling, Y., O'Gorman, J., Qian, F., Arastu, M., Li, M., Chollate, S., Brennan, M. S., Quintero-Monzon, O., Scannevin, R. H., Arnold, H., ... Sandrock, A. (2016). The antibody aducanumab reduces A $\beta$ plaques in Alzheimer's disease. Nature, 537(7618), 50-56. https://doi.org/10.1038/nature19323

Siemers, E. (2015). Drug Development in AD: Point of View from the Industry. Journal of Prevention of Alzheimer's Disease, 2(4), 216-218. https://doi.org/10.14283/ipad.2015.80

Singh, S. K., Srivastav, S., Yadav, A. K., Srikrishna, S., \& Perry, G. (2016). Overview of Alzheimer's Disease and Some Therapeutic Approaches Targeting $A \beta$ by Using Several Synthetic and Herbal Compounds. Oxidative medicine and cellular longevity, 2016, 7361613-7361613. https://doi.org/10.1155/2016/7361613 